\title{
NICU Admission Rates for suspected Growth Restricted Fetuses Born at Full Term
}

\author{
Madison Dolan ${ }^{1}$. Geoffrey Gordon ${ }^{2}$ \\ ${ }^{1}$ Indiana University School of Medicine; ${ }_{2}$ Parkview Health, Parkview Physicians Group: Maternal-Fetal \\ Medicine
}

\section{Background and Hypothesis:}

Fetal growth restriction (FGR) is the second leading cause for infant morbidity and mortality behind preterm birth. It is a sonographic estimated fetal weight (EFW) below the 10th percentile for gestational age. Neonates born $<2500 \mathrm{~g}$ at full-term are classified as "low birth weight." In the United States, more than $8 \%$ of all births fit this classification. These fetuses are at increased risk for both short and long term complications. Traditionally delivery at a NICU capable facility is recommended with an expected birth weight $<2500 \mathrm{~g}$. There is little data to support this arbitrary cut-off. This study was designed to compare rates of NICU admission and short term complications for liveborn neonates at full-term. We hypothesize these rates will be increased in neonates with a birth weight $<2500 \mathrm{~g}$.

\section{Methods:}

This is a proposed retrospective chart review of liveborn neonates at full-term at a regional medical system between January 2013 - December 2020. We excluded women under the age of 18, twins, fetuses with genetic syndromes, anomalies, or abnormal Umbilical Artery Dopplers. Neonates were categorized into two categories: birth weight between $2000-2499 \mathrm{~g}$ and birth weight between 2500-3000g. Outcomes included rates of NICU admission, tachypnea, acidosis, hypoglycemia, hyperbilirubinemia, feeding difficulties, APGAR scores, and temperature instability. Chi-square and logistic regression were used.

\section{Results:}

This study design will be submitted in the form of an IRB protocol. Results will include statistical comparisons between weight groups for NICU admission and other short term complications. Additionally data analysis on social and demographic characteristics will be done.

\section{Conclusions:}

Results of this study will help determine an adequate cut off for delivery planning and identify characteristics of the mother and fetus to be taken into consideration. These findings could increase need for a prospective trial, be used in evaluation of ultrasound accuracy, and impact the standard of care. 